Rev. Elev. Méd, Vét. Pays trop., 1967, 20, 3 (497-500).

\title{
Note sur la piroplasmose équine à Madagascar
}

\author{
par G. UILENBERG
}

\begin{abstract}
RÉSUMÉ
Cinq cas de piroplasmose équine sur des chevaux dont au moins 3 sont nés à Madagascar, due à Achromaticus equi (LAVERAN, 1901), ont été observés en 1965, et 1966 ; auparavant la piroplasmose équine n'avait été signalée qu'en 1903 (espèce en cause inconnue) et en 1950 (par A. equi). Un âne, né à Madagascar, s'est montré être porteur après splénectomie ; il est mart à la rechute malgré des traiternents au Pirodia (N.D.), à la Gonacrine (N.D.) et au Zothélone (N. D.); aucun piroplasmicide ne semble très actif sur $A$. equi. Un cheval, né sur place, s'est montré réfractaıre à l'injection de sang infectieux. Le vecteur à Madagascar reste inconnu; il ne peut s'agir, dans les cas observés, que d'une des tiques Amblyommo variegatum, Boophilus microplus ou Otobius megnin, mais aucune des trois n'est signalée comme vecteur dans la bıbliographie.
\end{abstract}

\section{INTRODUCTION}

La piroplasmose équine a été signalée à Madagascar pour la première fois par THIROUX en 1903. L'auteur ne dit pas s'il s'agit de chevaux importés ou nés sur place. A cette époque, une seule espèce des Babesiidae équınes était connue, Piroplasma equi Laveran, $1901=$ Nuttallia equi $=$ Babesio equi $=$ Achromaticus equi* ; ce n'est que plus tard qu'une deuxıème espèce fut distınguée, Babesia caball (Nuttall et Strickland, 1910). THIROUX présente les parasites comme des petits corps arrondis, les uns libres, les autres intraglobulaires, et il ajoute que LAVERAN a examiné ses préparations et a reconnu le parasite décrit par lui. II semble donc qu'il s'agissait de la petite espèce, $A$. equi; mais BUCK (1940) signale que THIROUX luı a fait savair que les parasites trouvés en 1903 se rapportent à la

(*) Nous avons exposé ailleurs (Uilenberg, sous presse) les raisons pour lesquelles nous considérons Achromaticus Dionisi comme nom générique correct pour les Babesiidae dant la multiplication dans les érythrocytes se fail habituellement par division en quatre. grande espèce, $B$. caballi, affirmation en contradiction avec sa description de 1903. II n'est donc pas possible de savoir avec certitude à quelle espèce THIROUX avait affare, non plus que de savorr s'il s'agit de cas autochtones ou importés.

Aucune autre mention de la piroplasmose équine jusqu'à 1940, quand BUCK trouve $B$. caballi et $A$. equi sur des mulets importés d'Algérie.

Ce n'est qu'en 1950 que le premier cas incontestablement contracté à Madagascar a été trouvé (BUCK et RAMAMBAZAFY, 1950); la description des parasıtes ne lasse aucun doute : il s'agit d'A. equi.

Depuis 1950 la piroplasmose équine n'a plus été signalée. Nous nous proposons de présenter quelques observations récentes.

\section{OBSERVATIONS PERSONNELLES}

1 - Un cheval de la Gendarmerie à Tananarive meurt le 13 septembre 1965 ; le responsable de l'animal déclare qu'il maigrissait depuis 4 ou 5 jours, et que l'urine avait, vers la fin, une 
couleur de café. II s'agit d'un cheval de 19 ans, importé de France vers 1952, resté à Tananarive depuis son arrivée. L'autopsie effectuée au Laboratoire quelques heures après la mort révèle une rate énorme $(12 \mathrm{~kg}$, longueur $72 \mathrm{~cm}$, largeur $44 \mathrm{~cm}$ ), un cœur «cuit», des hémorragies de la plèvre, des pétéchies sur les reins et de l'ictère.

Les frottis de sang et les calques de la rate, le foie et les reıns mettent en évidence d'assez nombreux A. equi.

Aucune tique n'est trouvée sur l'animal, mais Il est connu que les écuries de la Gendarmerie sont infestées par l'Argasıdé Otobius megninı.

Un $\mathrm{cm}^{3}$ environ de la rate est broyé dans de l'eau physiologique et mélangé à environ $1 \mathrm{ml}$ de sang récupéré dans une grande veine; ce mélange est inoculé aussitôt par vole intramusculaire à un cheval du Laboratoire (né à Madagascar), avec un résultat négatif (observation de la température et des frottis de sang pendant 3 semaines, observation clinique pendant un an.

20 7 janvier 1966. Des frottis de sang d'un cheval malade de la Gendarmerie à Tananarive, ayant une température de 40,50 , montrent de très rares A. equi. L'animal est né à Madagascar. Le Vétérinaire responsable injecte $1 \mathrm{~g}$ de Gonacrine (N. D.) (= Acriflavine) par vole intraveineuse, et signale une guérison rapide; il n'a trouvé aucune tique.

30 10 janvier 1966. Des frottıs de sang d'un autre cheval malade de la Gendarmerie à Tananarive, avec une température de $39,5^{\circ}$, montrent de très rares $A$. equi. Le Vétérinaire tratant signale que $1 \mathrm{~g}$ de Gonacrine amène de nouveau une guérison rapide; aucune tique n'est trouvée. Cet anımal est également né dans le pays.

4010 mai 1966. Des frottıs de sang sont reçus d'un poulain de la banlieve de Tananarive où Il est né ; les commémoratifs signalent une température de $39,1^{\circ}$ et une parésie du train arrière, l'animal ne pouvant se tenir debout.

Les frottis montrent de très rares $A$. equi.

Le Vétérinarre traitant injecte du Pirodia. (N. D.) (= Amicarbalıde) à la dose de $10 \mathrm{mg} / \mathrm{kg}$. Les parasites persistent dans les frottis faits le lendemain, et on signale une température de 39,30 , avec persistance de la parésie. Le Vétérinaire responsable injecte alors $0,5 \mathrm{~g}$ de Gonacrine, l'animal pesant $120 \mathrm{~kg}$. Les parasıtes ne sont plus trouvés sur frottis le 12 mai, la température est descendue à $38,5^{\circ}$, mais la parésie persiste. Nous avons appris par la suite que l'animal se mettait debout à partir du 15 mai ef qu'il est guéri.

Les seules tiques trouvées sur l'animal (et sur les autres chevaux de l'écurie) sont des nymphes d'O. megnini.

50 2 septembre 1966. Les frottis de sang d'un cheval malade à Tananarive montrent de très rares $A$. equi. Aucune tique n'est trouvée par le Vétérinaire traitant, qui guérit l'animal par la Gonacrine.

6o Un âne adulte est acheté à Soavinandriana, à environ $100 \mathrm{~km}$ au Sud-Ouest de Tananarive ; Il y est né. L'examen sur place montre qu'il est fortement infesté par des larves d'Anjlyomma varlegatum; aucune autre tique n'est trouvée. L'anımal est transporté au laboratore à Tananarive le 1er août 1966. II est splénectomssé le 18 octobre 1966, sulvant la méthode de DENINIG ef BROCKLESBY (1965).

Les premiers parasıtes, $A$. equi, sont observés dans son sang 3 jours plus tard. Leur nombre augmente rapidement pour atteindre approximativement 35 à 40 p. 100 d'érythrocytes infestés au be jour ; la température, restée en dessous de $38^{\circ}$ jusqu'alors, s'élève à $39,1^{\circ}$. Le Pirodıa à $10 \mathrm{mg} / \mathrm{kg}$, par voie intramusculaıre, n'empêche pas la parasitémie de monter à approximativement 60 p. 100 au $7 \mathrm{e}$ jour, pendant que la température augmente à $39,5^{\circ}$; il y a un ictère très intense mais l'appétıt reste normal. L'animal, pesant $230 \mathrm{~kg}$, reçoit alors, au $7 \mathrm{e}$ jour, $1 \mathrm{~g}$ de Gonacrine par voie intraveineuse. Le lendemain, le 8 e jour après l'opération, la parasitémie est restée stationnaire (environ $60 \mathrm{p}$. 100) ef les parasıtes gardent une morphologie normale ; la température est descendue à $38,5^{\circ}$, l'animal est un peu abattu. II est traité ce même jour au Zothélone (N.D.) (= Sulfate de quinuronium), à $1,2 \mathrm{mg} / \mathrm{kg}$ par voie saus-cutanée, administré en deux fois, avec un intervalle de 4 heures environ, ce qui n'amène pas de résultat appréciable non plus. La parasitémie diminue lentement pendant les jours suivants, pour aboutir au $12 \mathrm{e}$ jour à environ 30 p. 100 d'érythrocytes infestés; la morphologie des parasites change peu à peu, en ce sens que les formes anaplasmoides augmentent à partir du $9 \mathrm{e}$ jour, et elles 
constituent plus de 50 p. 100 du nombre de parasites ou $12^{\mathrm{e}}$ jour; les autres parasites ont un aspect normal. L'ictère persiste, ainsi que l'abattement; à aucun mament nous n'avons observé de l'hémoglobinurie. L'âne est trouvé mort le $13 \mathrm{e}$ jour après la splénectomie.

Les seules lésions observées à l'autopsie sont de l'ictère et de l'anémie. Les frottis des divers organes montrent de très nombreux $A$. equi dans les érythrocytes du cœur, du foie et des poumons ; ils sont très rares dans le rein, et un examen prolongé des capillaires du cortex cérébral n'en met pas du tout en évidence.

Le cheval du Laboratoire déjà utilısé pour l'essai de transmission d'A. equi (cas mortel de la Gendarmerie en 1965) est inoculé par voie sous-cutanée avec $10 \mathrm{ml}$ de sang de l'âne au 3 e jour après la splénectomie, contenant au mons 50 millions de parasites.

La température du cheval ne dépasse pas $38^{\circ}$ pendant le mois suivant, et les frottis de sang restent négatifs; l'animal ne montre aucun symptôme de maladıe par la suite.

\section{DISCUSSION ET CONCLUSIONS}

Les cas de piroplasmose équine sur des animaux dont il est certain qu'ils sont nés à Madagascar, ont tous été cousés par l'espèce $A$. equi.

L'infection par ce parasite pourrait être plus répandue que ne le feraient penser les longues périodes entre 1903 et 1950 et entre 1950 et 1965, pendant lesquelles aucun cas n'a été observé. En effet, le seul animal splénectomisé s'est montré être porteur et le seul animal inoculé avec du sang infectieux s'est montré être réfractaire, donc vraisemblablement prémuni.

Les essais sur l'âne splénectomisé montrent que le Pirodia à $10 \mathrm{mg} / \mathrm{kg}$ est sans effet sur A. equi, observation confirmée por l'échec de ce traitement sur le cas du poulain. Nous avons été surpris, étant donné les bons résultats dans la pratique, que la Gonacrine n'ait pas non plus montré d'activité sur les parasıtes de l'âne. II est impossible de dire si le Zothélone a eu une action quelconque; la parasitémie a certes lentement diminué après ce traitement, les formes anaplasmoides ont augmenté et la température est devenue normale, mais cela peut être dû à l'effet accumulé des trois traıtements; l'actıvité éventuel du Zothélone est tout au plus minime. (YAKIMOFF (1940) signale d'alleurs que les formes anaplasmoïdes apparaissent quand la guérison commence, soit à la suite de traitement soit par le développement d'anticorps). DENNIG (1965) ne voit aucun effet du traitement par l'Accoprine (N. D.), dont le Zothélone est un équivalent, sur l'évolution de la rechute chez les ânes splénectomısés; if ne voit pas d'effet non plus du Berenil (N.D.), nı de la Phenamidine. Aucun prroplasmicide ne semble avoir une action spécifique et rapide sur $A$. equi.

Quel est le vecteur à Madagascar? Dans la région de Tananarive il n'existe que trois espèces de tiques pouvant attaquer les chevaux: Amblyommo variegatum, Boophilus microplus et Otobus megnini; A. variegatum y est rare, les deux autres espèces sont très communes. Dans la régıon de Socvinandriana, d'où venaıt l'âne, A. variegatum et $B$. microplus existent, $O$. megnin n'y semble pas encore implanté. Ailleurs, dans les régions côtıères de Madagascar, l'espèce Rhipicephalus sanguneus est trouvée. II n'existe pas d'autres tiques dans le pays, susceptıbles de s'attaquer aux chevaux; la seule de ces quatre espèces pour laquelle la transmission expérimentale d'A. equi ait été signalée est Rh. sanguineus, mais elle peut être exclue dans les cas de la région tananarivienne où elle n'existe pas; elle ne semble pas non plus avoir été trouvée dans la région de Socvinandrianc. Les possibilités sont donc limitées aux trois autres espèces, qui toutes ont été trouvées sur le cheval à Madagascar, mais pour lesquelles la transmission d'A. equr n'est pas signalée dans la bibliographie. Seules des expériences pourront décıder.

Instifut d'Elevage et de Médecine Vétérinaire des Poys Tropicaux.

Laboratorre Central de l'Elevage de Tonanarive (Service d'Entomologie ef de Protozoologie). 


\section{SUMMARY}

Note on equine piroplasmosis in Madagascar

Five cases of equine piroplasmosis due to Achromaticus equi (LAVERAN, 1901). have been seen during 1965 and 1966 in horses of which al least 3 were born in Madagascar ; equine piraplasmosis had only been reported before in 1903 (causal species unknown) and in 1950 (by A. equi). A donkey, born in Madagascar, was shown to be a carrier of $A$. equ by splenectomy; it died by the relapse, in spite of treatment with Pirodia R, Gonacrine R and Zothelone R ; no piroplasmicidal drug seems to be very active against $A$. equi. An indigenous horse was refractory to injection with infected blood. The vector in Madagascar remains unknown; it can only be, in the cases seen, one of the ticks Ambiyomma variegotum, Boophilus microplus or Otobius megnini, but none of these is reported as a vector in the literature.

\section{RESUMEN}

Nota sobre la piroplasmosis de los caballos en Madagascar

En 1965 y 1966, se observaron cinco casos de piroplasmosis causada por Achroma, ticus equi (LAVERAN, 1901) en caballos de los cuales 3 por lo menos nacie ronen Madagascar.

Antes, no se habia notado la piroplasmosis sino en 1903 (causada por una especie desconocida) y en 1950 (por A. equi). Un burro, nacıdo en Madagascar, estuvo portador después de la esplenectomıa; murió durante la recaida a pesar de los tratamientos con Pirodia (N. D.), Gonacrine (N. D.) y Zothelone (N. D.). Ningún prroplasmıcido parece muy activo contra $A$. equi. Un caballo, nacido en el pais, se mostró refractario a la inyección de sangre infecciosa. El vector queda desconocido en Madagascar ; En los casos notados, solo se trata verosimilmente de una de las garrapatas Ambiyomma variegatum, Boophilus microplus u Otobius megntni, pero se nota ninguna de las tres como vector en la bibliografía.

\section{BIBLIOGRAPHIE}

1. BUCK (G.). - A propos des piroplasmoses des équidés à Madagascar. Bull. Soc. Path. exot., 1940, 33, 86-89.

2. BUCK (G.) et RAMAMBAZAFY. - Premier cas de nuttaliose naturelle signalée à Madagascar. Bull. Soc. Path. exot., 1950, 43, 43-44.

3. DENNIG (H. K.). - Die Beeinflussung der lafenten Pferdepiroplasmose (Babesia equi Laveran) durch die Splenektomie. Berl. Munch. tierârztl. Wschr., 1965, 78, 204-209.

4. DENNIG (H. K.) et BROCKLESBY (D.W.). - Splenectomy of horses and donkeys. Vet. Rec., 1965, 77, 40-44.
5. THIROUX (M.). - Note sur l'existence de la piroplasmose du cheval à Madagascar. C. R. Soc. Biol., Paris, 1903, 55, 1188-1189.

6. UILENBERG (G.). - Trois nouveaux parasites d'insectivores malgaches : Achromaticus brygooi sp. n. (Sporozod, Babesiidae), Eimeria setosi sp. n. et Eimeria madagascariensis sp. n. (Sporozod, Eimeriidae). Ann. Porosit. hum. comp. (sous presse).

7. YAKIMOFF (W. L.). - (Formes anaplasmoïdes dans la nuttalliose équine). Veterinariya, Moscou, 1940 (5), 56-59 (Extrait dans Vet. Bull., 1946, 16, 140). 